\title{
Functional connectivity alterations in the motor and fronto-parietal network relate to behavioral heterogeneity in Parkinson's disease
}

\author{
Authors: \\ Griet Vervoort ${ }^{1}$, MSc, griet.vervoort@faber.kuleuven.be \\ Kaat Alaerts ${ }^{1}$, Prof., kaat.alaerts@faber.kuleuven.be \\ Aniek Bengevoord ${ }^{1}$, MSc, aniek.bengevoord@faber.kuleuven.be \\ Evelien Nackaerts ${ }^{1}$, MSc, evelien.nackaerts@faber.kuleuven.be \\ Elke Heremans ${ }^{1}$, PhD, elke.heremans@ faber.kuleuven.be \\ Wim Vandenberghe ${ }^{2}$, Prof., wim.vandenberghe@uzleuven.be \\ Alice Nieuwboer ${ }^{1}$, Prof., alice.nieuwboer@ faber.kuleuven.be: Corresponding author
}

\begin{abstract}
Affiliations:
${ }^{1}$ KU Leuven, Department of Rehabilitation Sciences, Tervuursevest 101/1501, 3001 Leuven, Belgium

${ }^{2}$ University Hospitals Leuven, Department of Neurology, Herestraat 49, 3000 Leuven, Belgium
\end{abstract}

Key words: Parkinson's disease, resting-state fMRI, subtypes, heterogeneity, motor control 


\begin{abstract}
Background: Insight into the neural mechanisms of postural instability and gait disorder (PIGD) and tremor dominant (TD) subtypes in Parkinson's disease (PD) is indispensable for generating pathophysiology hypotheses underlying this phenotyping. This cross-sectional study aimed to gain insight in specific and brain-wide functional connectivity (FC) and its correlation with motor deterioration and preservation in PD subtypes.
\end{abstract}

Methods: 68 PD patients classified as PIGD $(n=41)$, TD $(n=19)$ or indeterminate $(n=8)$ and 19 agematched controls underwent resting-state fMRI while 'off' medication to assess FC between regions of interest (ROIs) in the motor and fronto-parietal network and on a whole-brain level using a parcellated template. FC alterations were correlated with quantitative behavioral measures.

Results: ROI-analyses showed decreased FC between the caudate and putamen in PIGD compared to TD. This hypo-connectivity was correlated with behavioral impairment. In contrast, TD-specific hyper-connectivity between motor cortical areas and the inferior parietal lobule correlated with less behavioral impairment, suggesting compensatory mechanisms. Both subgroups showed hyperconnectivity between the left supplementary motor area and pedunculopontine nucleus, whereas PIGD-specific right lateralized hyper-connectivity was shown between this nucleus and the premotor cortex. Whole-brain analyses revealed 65\% hypo-connectivity and 35\% hyper-connectivity in PIGD compared to TD. TD also revealed primarily hypo-connectivity compared to controls, but had more pronounced hyper-connectivity involving temporo-occipital areas.

Conclusion: This multilevel analysis showed differential connectivity alterations in large scale neural networks and between motor and cognitive control areas that related to behavioral heterogeneity in PD, underscoring the classic TD-PIGD phenotypical classification. 


\section{INTRODUCTION}

Clinical phenotypes of Parkinson's disease (PD), i.e. the Postural Instability and Gait Disorder (PIGD) and Tremor Dominant (TD) phenotype, are distinguished by a spectrum of motor and nonmotor symptoms[1], suggesting different underlying neural mechanisms[2-4], Previous studies predominantly focused on the striatal loss of dopamine transporters[5, 6] or on areas of the default mode network (such as the dorsolateral prefrontal cortex, precuneus and inferior parietal lobule)[4, 7] to characterize the neural basis of PD heterogeneity. However, mapping functional connectivity (FC) alterations underlying the specific symptom patterns in PD subtypes requires a broader analysis of motor control and fronto-parietal networks[8]. To date, this approach was not yet applied.

Therefore, we analyzed specific regions of interest (ROI) within networks that have been implicated in freezing of gait (FOG)[9], a prominent symptom in PIGD and not in TD. Previous imaging studies often lacked detailed behavioral information other than Unified Parkinson's Disease Rating Scale (UPDRS) scores[4]. To understand whether pathological or compensatory mechanisms underpinned behavioral differences, we correlated FC measures with cognitive outcomes and quantitative gait and balance measures known to be sensitive subtype discriminators $[2,10]$.

Although PD symptomatology suggests predominant involvement of fronto-striatal and frontoparietal regions, it is unclear if other networks are also implicated in subtype pathophysiology. In this context, whole-brain studies showed increased gray matter atrophy in all major cortical areas in PIGD, whereas tremor was related to cerebellar gray matter decreases and cerebello-thalamo-cortical circuit disruption $[3,4,11]$. Again, none of these studies investigated FC at a brain-wide level. Therefore, in a complementary analysis we aimed to clarify the extent of FC differences at the neurological systems level. The current study innovatively mapped whole brain FC in PD subtypes using a parcellated template that utilizes functionally coherent parcellations while overcoming the disadvantages inherent to data-driven clustering methods[12].

By combining a hypothesis-driven approach with behavioral correlations and a whole-brain analysis, this study aimed to significantly enhance the understanding of the neurological mechanisms underpinning behavioral heterogeneity in PD, which is a crucial step in developing tailored treatment. Due to the suggested relationship between FOG and PIGD [13], we hypothesized that more severely disturbed FC would be present in PIGD compared to TD and healthy controls in the motor and cognitive control network [14]. We particularly expected altered FC involving the striatum, supplementary motor area (SMA) and pedunculopontine nucleus (PPN) as reduced activity in these areas was previously shown to correlate with lower gait speed[15] and postural instability[16].We 
also expected that greater connectivity in TD versus PIGD and controls would underlie better motor scores, possibly involving primary and secondary motor areas $[8,17]$.

\section{METHODS}

\section{Participants}

Eighty-six early to mid-stage PD patients and 19 age-matched controls were included (for details, see Supplementary Materials). Patients were classified as PIGD $(n=41)$, TD $(n=19)$ or indeterminate $(\mathrm{n}=8)$ based on sub-scores of the MDS-UPDRS while "off" medication (i.e. 12 hours after last medication intake)[1, 18]. Patient subgroups were a-priori group-matched for age and disease severity (MDS-UPDRS III) to account for disease-confounding effects. Patients were included if diagnosed with PD based on UK brain bank criteria. Participants were excluded if they had a MiniMental State Examination (MMSE) score < 24 and/or presented with neurological comorbidities or MRI contra-indications. Indeterminate patients were excluded from further analyses. All participants underwent cognitive assessment and quantitatively measured motor and balance tests (MiniBESTest) in our 3D motion analysis lab while "off” medication in the context of a previous study[10] (for details, see Supplementary Materials). The outcome measures that were significantly different between PIGD and TD were included in the correlation analysis of the current study. The study was approved by the local ethics committee of the University Hospitals Leuven and all patients gave written informed consent according to the declaration of Helsinki.

\section{Image acquisition}

All subjects underwent a rs-fMRI scan while "off” medication (Philips 3T ACHIEVA MRI scanner (Best, The Netherlands)) using a T2*-weighted Gradient Echo Planar Imaging (FEEPI) sequence (duration: 435s, slice number: 31 ; slice thickness: $4 \mathrm{~mm}$; repetition time (TR): $1.7 \mathrm{~s}$; echo time (TE): 33ms; flip angle: 90; matrix: 64x64; FOV: 230x124x230mm, voxel size: 3.59x3.74x4mm; $3 \times 3 \times 3 \mathrm{~mm}$ after normalization[19]) in combination with a high-resolution anatomical T1-weighted sequence (T1 Turbo Field Echo (TFE) sequence, duration: 383ms; slice number: 182; slice thickness: 1.2mm; TR: 9.624s; TE: 4.6ms; flip angle: $8^{\circ}$; matrix: 256x256; FOV: 218.4x250x250mm). 


\section{Image processing}

All images were visually inspected for large image artifacts. Next, rs-fMRI images without visually noticeable artifacts underwent pre-processing using SPM8 (Wellcome Department of Imaging Neuroscience, University College London, UK) implemented in Matlab® R2011b. All functional images were realigned to the reference (mean) image and slice-time corrected. The realigned images were co-registered to each subject's T1 anatomical image, which were segmented to generate a white matter, gray matter and cerebrospinal fluid mask[20]. All images were normalized to Montreal Neurological Institute (MNI) space and smoothed with a $5 \mathrm{~mm}$ full width at half maximum Gaussian kernel[19]. The first 4 volumes of the functional scan were omitted to account for saturation effects. First and second level analyses were performed using the CONN toolbox (v.14) (http://www.nitrc.org/projects/conn, see Supplementary Materials). All rs-fMRI images were motioncorrected using six translational and rotational realignment parameters. As an additional motion correction, we accounted for instantaneous head motion by calculating frame-wise displacement (FD) for each volume. This accounts for the frame-wise change in brain image intensity based on the (Blood Oxygenation Level Dependent) BOLD response. If the intensity changed more than $0.5 \%$ from one volume to the next ( $\mathrm{FD}>0.5 \mathrm{~mm})$, this volume was censored. All analyses were performed on 'scrubbed' data, i.e. without the censored volumes. If the proportion of censored volumes exceeded $50 \%$, subjects were excluded from the analysis.

\section{ROI-to-ROI analysis}

We explored FC for core areas of the motor and fronto-parietal network (Table S1) [21, 22]. Subcortical ROI coordinates and sizes were based on previous work from our lab[20] and cortical ROIs [21, 22] were selected from previous studies on freezing of gait (FOG) [9]. Cortical and cerebellar ROIs were constructed as spheres with $8 \mathrm{~mm}$ radius [23] and the SNc was constructed with a radius of $3 \mathrm{~mm}$ around the center coordinates as in $\mathrm{Wu}$ et al.[21]. All ROIs were co-registered to each subject's structural and functional images. Time series for the defined ROIs were Pearson correlated to all other ROIs. Between-group differences were tested using ANCOVA's with age, disease duration and severity as covariates and false discovery rate (FDR) corrected p-values [24] with $\alpha=0.05$. The FDR is the proportion of false positives among those tests for which the null hypothesis is rejected [25]. Because Levodopa Equivalent Dose (LED) was significantly correlated 
with disease duration $(\mathrm{rho}=0.42 ; \mathrm{p}=0.001)$ and all scans were performed 'off' medication, we chose not to include LED as a covariate.

\section{Behavioral Correlations}

Differential FC indices between groups were correlated with clinical measures (PIGD score, TD score, Mini-BESTest, stride length, UL dyscoordination, LL amplitude, MMSE, TMT-A, TMT-B and FAB) across all patients using Spearman correlations which were Bonferroni corrected for multiple testing ( $\alpha=0.005$ ). Correlations with rho $<-0.3$ or $>0.3$ were considered. Behavioral outcomes were selected due to their discriminatory ability between PD subgroups as determined in earlier work[10].

\section{Parcellated whole-brain analysis}

To map whole-brain FC, a template dividing the brain into 200 parcellations based on functional and structural coherence was used[12]. These regions were entered as ROIs in CONN (v.14) (see Supplementary Materials). Subsequent analyses were performed analogously to the 'ROI-to-ROI analysis'. To allow interpretation within a framework of known anatomical boundaries, parcellations were overlaid with the Automated Anatomical Labeling (AAL) atlas. This information was used to construct FC matrices (see Supplementary Materials).

\section{Statistics for subject characteristics}

Subject characteristics and FD were compared between patient groups using unpaired t-tests or Mann-Whitney $U$ tests depending on the distributions. Categorical variables were compared using Chi-square tests. Comparisons between 3 groups were done using ANOVAs with post-hoc TukeyHSD tests or Kruskall-Wallis ANOVAs with post-hoc Mann-Whitney U tests depending on the distribution (Statistica v.10 Stat Soft. Inc). 


\section{RESULTS}

\section{Subject characteristics}

All subgroups were comparable for age, sex and disease severity (Table 1). PIGD had longer disease duration, higher LED, higher PIGD-scores and lower TD-scores compared to TD. Eight PIGD and no TD patients presented with FOG. PIGD had worse balance (mini-BESTest) and shorter stride lengths compared to TD and controls. UL dyscoordination and LL amplitude were more impaired in PIGD compared to controls. PIGD scored worse on the MMSE and Trail Making Test (TMT)-B compared to TD and worse on TMT-A compared to controls. Frontal Assessment Battery (FAB)-scores were lower in both subgroups than controls. Mean framewise displacement (FD) was not significantly different between groups (Figure S1).

Insert Table 1 about here

\section{ROI analysis of motor and fronto-parietal network connectivity}

Three (2 PIGD and 1 TD) patients and 1 control were excluded from the resting-state analysis due to image artifacts. In addition, FD-correction led to exclusion of 6 PIGD and 2 TD, bringing the final sample size to 18 controls, 33 PIGD and 16 TD.

Figure 1 provides a schematic overview of the direct comparison between PIGD and TD. ANCOVA analyses showed decreased FC in PIGD compared to TD within the striatum, specifically between the left caudate and bilateral ventral and dorsal putamen (Table 2). PIGD additionally showed lower FC between the left caudate and right pallidum and between the left caudate and the left PMC and right superior temporal lobe. We found no increased FC in PIGD compared to TD.

In the same vain, PIGD showed predominantly subcortical hypo-connectivity with decreased FC within the striatum, between the pallidum and putamen and between sub-regions of the thalamus compared to controls (Table 2). We also found decreased FC between the left thalamus (part projecting to M1 (thal-M1)) and the contralateral precuneus and ipsilateral dorsal putamen, substantia nigra pars compacta (SNc), subthalamic nucleus (STN) and insula. Additionally, we found decreased FC between the left dorsal putamen and supplementary motor area (SMA)-proper. The left 
pedunculopontine nucleus (PPN), in contrast, showed increased FC with the SMA-proper, while the right PPN was hyper-connected to the right PMC and left M1.

Insert Table 2 and Figure 1 about here

The TD-control comparison showed less hypo-connectivity and more pronounced hyper-connectivity than the PIGD-control comparison (Table 2). Particularly, decreased FC within the thal-PMC and increased FC between the left PPN and the left PMC, pre-SMA and SMA-proper was revealed. In addition, the right inferior parietal lobule (IPL) showed increased FC with the bilateral M1 and PMC in TD compared to controls.

\section{Behavioral Correlations}

Spearman correlation in the entire patient group $(n=68)$ showed that both lower PIGD - and higher Mini-BESTest-scores correlated with increased FC between the right IPL and the right PMC and M1 (Figure S2). Worse balance was additionally correlated to reduced FC between the right caudate and left dorsal putamen, while decreased FC within the ventral putamen and between the bilateral and dorsal ventral putamen were associated with worse UL coordination (Table 3). At the cognitive level, decreased FC between the left caudate and left ventral and dorsal putamen was related to lower MMSE-scores. A similar pattern was found for longer TMT-B completion times, which were additionally correlated with reduced FC within and between the dorsal and ventral putamen as well as with reduced FC between the right caudate and the bilateral dorsal putamen and between the bilateral pallidum and the ipsilateral dorsal putamen. In contrast, longer TMT-A completion times were only associated with decreased FC within thal-M1. Lower FAB-scores were correlated with decreased FC within the dorsal putamen and between the right caudate and left dorsal putamen. No correlations were found with TD-scores, stride length or LL amplitudes. 


\section{Parcellated whole-brain analysis}

Direct comparison of FC patterns in PIGD $(n=33)$ relative to TD $(n=16)$ (Figure 2a) using ANCOVA analyses showed both reduced (65\%) and increased FC (35\%). FC reductions were most pronounced between the middle frontal gyrus and the precuneus, fusiform gyrus, parahippocampal gyrus and the cerebellum as well as within the temporal lobe (Figure S3). The pattern of increased FC was dominated by connections between the thalamus and occipito-temporal areas and between cingulate and frontal areas.

Insert Figure 2 about here

Figure $2 \mathrm{~b}$ shows a predominant pattern of hypo-connectivity in PIGD ( $\mathrm{n}=33)$ compared to controls $(n=18)$ : $97 \%$ of all identified connections were hypo-connections, while only $3 \%$ were hyperconnections. Overlaying parcellations with the AAL atlas (Figure S4) revealed a large number of hypo-connections within the frontal and temporal lobe in PIGD compared to controls. In addition, connections between the primary somatosensory cortex (S1) and the occipital and fusiform gyrus in the temporal lobe and connections within the striatum contributed significantly to the hypoconnectivity pattern. Hyper-connectivity (3\%) in PIGD was located between the paracentral lobule and middle frontal gyrus and between the brainstem and insula.

Comparison of FC between TD $(n=16)$ and controls $(n=18)$ revealed a predominant pattern of hypoconnectivity (84.2\%), as well as several hyper-connections (15.8\%) (Figure 2c). A large number of the hypo-connections was situated between the middle temporal gyrus and several areas in the frontal lobe, between the insula and S1 and between the postcentral gyrus (rolandic region) and occipital lobe areas (Figure S5). While PIGD showed decreased FC between temporal lobe and occipital areas compared to controls, the TD group showed increased FC between the middle temporal gyrus and occipito-temporal areas. Furthermore, the occipito-temporal areas showed hyper-connectivity with the precentral gyrus in TD compared to controls. 


\section{DISCUSSION}

In this study, we used two complementary rs-fMRI methods to investigate connectivity patterns in PIGD, TD and controls while controlling for subgroup differences. The results show differential neural network involvement in PD subtypes, a pattern which is strengthened by correlations to behavioral outcomes in the motor and cognitive domain. In the following paragraphs we will discuss the neuroimaging results that were specific to either the PIGD or TD subgroup. First, we will focus on the ROI-based results including their behavioral correlations. Next, we will discuss subgroupspecific changes in the brain-wide connectome.

\section{ROI analysis of motor and fronto-parietal network connectivity}

Confirming our hypothesis, the ROI-analysis showed clear hypo-connectivity in the motor and fronto-parietal network, specifically within the striatum in PIGD compared to TD and controls. Decreased FC involving the caudate may indicate more widespread dopaminergic functional loss in PIGD underlying more severe behavioral impairment, as it associated with worse balance and more impaired cognition. Earlier studies endorse this finding by showing lower striatal density of dopamine transporters in PIGD compared to TD[6].

Previous studies in PD, irrespective of subtype, suggested that dopaminergic functional loss is compensated for by increased FC between the anterior putamen and cortical motor areas[26, 27]. In contrast, we found decreased FC between the dorsal putamen and SMA-proper in PIGD compared to controls. Reduced activity and connectivity involving the putamen and SMA has been the most consistent finding in PD neuroimaging studies, implicated in the maintenance of stride amplitude during gait and FOG[28, 29]. Given these earlier findings, our results may point to a pure motor explanation of why PIGD have more gait deficits than TD. TD on the other hand, seemed to rely on primary and secondary motor areas to boost motor function as was corroborated by hyperconnectivity between these areas and the IPL. As the IPL is involved in monitoring motor function based on visuoperceptual information[30] and its increased FC proved to be predictive of better balance, it likely signifies a compensatory mechanism via bottom-up orientation of attention.

Next to the specific involvement of the striatum in PIGD, we found reduced FC between the striatum and pallidum in PIGD compared to TD and controls. Decreased pallidal dopamine levels[31] and task-related pallidal activity have been demonstrated in studies comparing TD with non-TD groups. 
The striatal-pallidal connection has a key inhibitory function within the direct and indirect basal ganglia pathways. Recently, it was suggested that both pathways are possibly integrated at the striatal and pallidal level, rather than being two separate entities[32]. The abundantly reduced PIGD-specific striatal connectivity could thus alter the balance between the direct and indirect pathways, partially explaining the more pronounced gait and balance problems within this subgroup. Moreover, decreased neuropsychological performance following pallidal Deep Brain Stimulation (DBS)[33] suggests that cognitive circuits are mediated by this region, Altered FC within these circuits could therefore account for PIGD-specific cognitive impairment. The correlation of reduced FC between the striatum and pallidum with higher TMT-B completion times supports this notion. As no such correlation was found with TMT-A scores, striato-pallidal hypo-connectivity in PIGD might specifically be involved with difficulties in set-shifting and executive functioning.

Furthermore, we demonstrated PIGD-specific hyper-connectivity between the right PPN and cortical motor areas. As right-lateralization of structural and functional connectivity alterations of the PPN has previously been associated with FOG[34], our results suggest that this mechanism might also play a role in PIGD. Fling et al. interpreted the hyper-connectivity between the right mesencephalic locomotor region and SMA to be maladaptive as it was correlated with more severe FOG[9]. Previous work has also shown that balance deficits and falls are associated with a decreased number of PPN cholinergic neurons in PD[16]. Future study combining structural and functional connectivity analysis may shed further light on the role of the PPN in PIGD.

\section{Parcellated whole-brain analysis}

Confirming the ROI-based analysis, both patient groups showed a brain-wide pattern of predominantly hypo-connectivity compared to controls. Hyper-connectivity, however, was more prominent in TD compared to controls, as was shown previously[35]. Direct subgroup comparison also revealed increased FC in 35\% of all identified connections in PIGD compared to TD. Other neuroimaging studies support distinct and widespread neural differences between PD subgroups, although they mostly focused on structural deficits[2, 3].

The results from the whole-brain analysis fit within the compensation model suggested by Helmich et al.[26] as we found a substantial amount of hyper-connectivity between the thalamus and the middle temporal gyrus and fusiform gyrus in PIGD versus TD. Deficient thalamo-cortical loops were previously suggested to be counterbalanced by enhanced cerebellar-cortical projections and the 
involvement of the precuneus[36]. Decreased FC between the frontal lobe and cerebellum as well as with the precuneus in our PIGD cohort may indicate that these compensatory mechanisms are less prominent in PIGD.

Supporting behavioral studies which demonstrated that visuoperceptual impairments contribute to FOG[37], our whole-brain analysis indicated PIGD-specific hypo-connectivity within visual occipital and temporal areas. As these areas were hyper-connected in TD compared to controls, this suggests up-regulated visuoperceptual pathways as an additional compensatory mechanism for motor control deficits. Our ROI-based results reinforced this finding as thal-M1 was less connected to the precuneus and insula in PIGD compared to controls. Since the insula controls sensorimotor integration during goal-directed movements via the thalamus[38], its hypo-connectivity could compromise goal-directed actions in PIGD and not in TD.

\section{Limitations}

Despite controlling for disease confounds, we cannot rule out that disease progression may have influenced our results. As the study was performed 'off' medication and LED correlated with disease duration, we opted not to correct for LED. To draw definite conclusions about the contribution of disease progression to PD phenotypes, longitudinal FC studies are indispensable. These studies would benefit from accounting for white matter disease and should consider applying smoothing procedures as signals could be blurred if small ROIs undergo smoothing with a smoothing kernel of a similar size. Moreover, as blood volume changes might influence the BOLD response[39], future studies could benefit from collecting information on hydration to optimize reliability of rs-fMRI data.

The current study applied FDR-correction to account for multiple testing. While this procedure is a valid approach deal with the large number of statistical tests inherent to neuroimaging analyses[25], it is not as stringent as Bonferroni corrections and therefore could have led to type I errors. Even though the sex distributions were not significantly different between groups, we cannot fully exclude that this may have biased our results as a recent resting-state fMRI study reported altered regional homogeneity in healthy young males compared to females[40]. Although we interpreted that the observed hyper-connectivity patterns are likely to be compensatory in nature, in line with our correlation analysis, it remains unclear if it truly represents functional compensation rather than nonspecific pathological activity[41]. However, compensatory mechanisms could be hypothesized to counterbalance the loss of dopaminergic signaling and therefore at least partially restore behavioral 
impairment caused by the primary anatomical deficit[41, 42]. To elucidate this, multimodal brain imaging studies are warranted.

\section{Conclusion}

Our results showed PIGD-specific hypo-connectivity primarily within the striatum and in important motor and cognitive control loops. Hyper-connectivity involving the PPN also seemed to contribute to PIGD-pathophysiology. TD-specific hyper-connectivity in visual areas, IPL and cortical motor areas rather pointed to mechanisms that may compensate for decreased movement automaticity. As such, the findings help to unravel the critical mechanisms underlying motor-cognitive phenotypes in PD.

\section{Acknowledgements}

We would like to thank all subjects for their motivated and enthusiastic participation in this study. Special thanks go to prof. Lynn Rochester for fruitful discussions in the course of this project. Support for this study was provided through a grant from the Special Research Fund of KU Leuven, Belgium (contract OT / 11/ 091), Malou-Malou funds of the King Baudouin foundation, Research Foundation Flanders project G.0906.11 and funding through the Gabe Foundation. All authors report no conflict of interest.

\section{References}

[1] Jankovic J, McDermott M, Carter J, Gauthier S, Goetz C, Golbe L, et al. Variable expression of Parkinson's disease: a base-line analysis of the DATATOP cohort. The Parkinson Study Group. Neurology. 1990;40:1529-34.

[2] Bohnen NI, Müller MLTM, Zarzhevsky N, Koeppe Ra, Bogan CW, Kilbourn MR, et al. Leucoaraiosis, nigrostriatal denervation and motor symptoms in Parkinson's disease. Brain : a journal of neurology. 2011;134:2358-65.

[3] Rosenberg-Katz K, Herman T, Jacob Y, Giladi N, Hendler T, Hausdorff JM. Gray matter atrophy distinguishes between Parkinson disease motor subtypes. Neurology. 2013;80:1476-84. 
[4] Zhang J, Wei L, Hu X, Xie B, Zhang Y, Wu GR, et al. Akinetic-rigid and tremor-dominant Parkinson's disease patients show different patterns of intrinsic brain activity. Parkinsonism Relat Disord. 2015;21:23-30. [5] Rossi C, Frosini D, Volterrani D, De Feo P, Unti E, Nicoletti V, et al. Differences in nigro-striatal impairment in clinical variants of early Parkinson's disease: evidence from a FP-CIT SPECT study. European journal of neurology : the official journal of the European Federation of Neurological Societies. 2010;17:62630.

[6] Eggers C, Pedrosa DJ, Kahraman D, Maier F, Lewis CJ, Fink GR, et al. Parkinson subtypes progress differently in clinical course and imaging pattern. PloS one. 2012;7:e46813-e.

[7] Prodoehl J, Planetta PJ, Kurani AS, Comella CL, Corcos DM, Vaillancourt DE. Differences in brain activation between tremor- and nontremor-dominant Parkinson disease. JAMA neurology. 2013;70:100-6.

[8] Baudrexel S, Witte T, Seifried C, von Wegner F, Beissner F, Klein JC, et al. Resting state fMRI reveals increased subthalamic nucleus-motor cortex connectivity in Parkinson's disease. NeuroImage. 2011;55:172838.

[9] Fling BW, Cohen RG, Mancini M, Carpenter SD, Fair DA, Nutt JG, et al. Functional reorganization of the locomotor network in Parkinson patients with freezing of gait. PLoS One. 2014;9:e100291.

[10] Vervoort G, Bengevoord A, Nackaerts E, Heremans E, Vandenberghe W, Nieuwboer A. Distal motor deficit contributions to postural instability and gait disorder in Parkinson's disease. Behav Brain Res. 2015;287:1-7.

[11] Helmich RC, Janssen MJ, Oyen WJ, Bloem BR, Toni I. Pallidal dysfunction drives a cerebellothalamic circuit into Parkinson tremor. Ann Neurol. 2011;69:269-81.

[12] Craddock RC, James GA, Holtzheimer PE, Hu XP, Mayberg HS. A whole brain fMRI atlas generated via spatially constrained spectral clustering. Hum Brain Mapp. 2012;33:1914-28.

[13] Factor Sa, Steenland NK, Higgins DS, Molho ES, Kay DM, Montimurro J, et al. Postural instability/gait disturbance in Parkinson's disease has distinct subtypes: an exploratory analysis. Journal of neurology, neurosurgery, and psychiatry. 2011;82:564-8.

[14] Tessitore A, Amboni M, Esposito F, Russo A, Picillo M, Marcuccio L, et al. Resting-state brain connectivity in patients with Parkinson's disease and freezing of gait. Parkinsonism Relat Disord. 2012;18:781-7.

[15] Peterson DS, Pickett Ka, Duncan RP, Perlmutter JS, Earhart GM. Brain activity during complex imagined gait tasks in Parkinson disease. Clinical neurophysiology : official journal of the International Federation of Clinical Neurophysiology. 2014;125:995-1005.

[16] Karachi C, Grabli D, Bernard Fa, Tandé D, Wattiez N, Belaid H, et al. Cholinergic mesencephalic neurons are involved in gait and postural disorders in Parkinson disease. The Journal of clinical investigation. 2010;120:2745-54. 
[17] Kurani AS, Seidler RD, Burciu RG, Comella CL, Corcos DM, Okun MS, et al. Subthalamic nucleussensorimotor cortex functional connectivity in de novo and moderate Parkinson's disease. Neurobiology of aging. 2014:1-8.

[18] Stebbins GT, Goetz CG, Burn DJ, Jankovic J, Khoo TK, Tilley BC. How to identify tremor dominant and postural instability/gait difficulty groups with the movement disorder society unified Parkinson's disease rating scale: comparison with the unified Parkinson's disease rating scale. Movement disorders : official journal of the Movement Disorder Society. 2013;28:668-70.

[19] Alaerts K, Geerlings F, Herremans L, Swinnen SP, Verhoeven J, Sunaert S, et al. Functional Organization of the Action Observation Network in Autism: A Graph Theory Approach. PLoS One. 2015;10:e0137020.

[20] Vercruysse S, Spildooren J, Heremans E, Wenderoth N, Swinnen SP, Vandenberghe W, et al. The Neural Correlates of Upper Limb Motor Blocks in Parkinson's Disease and Their Relation to Freezing of Gait. Cereb Cortex. 2014;24:3154-66.

[21] Wu T, Wang J, Wang C, Hallett M, Zang Y, Wu X, et al. Basal ganglia circuits changes in Parkinson's disease patients. Neurosci Lett. 2012;524:55-9.

[22] Postuma RB, Dagher A. Basal ganglia functional connectivity based on a meta-analysis of 126 positron emission tomography and functional magnetic resonance imaging publications. Cereb Cortex. 2006;16:150821.

[23] Snijders AH, Leunissen I, Bakker M, Overeem S, Helmich RC, Bloem BR, et al. Gait-related cerebral alterations in patients with Parkinson's disease with freezing of gait. Brain. 2011;134:59-72.

[24] Noble WS. How does multiple testing correction work? Nat Biotechnol. 2009;27:1135-7.

[25] Genovese CR, Lazar NA, Nichols T. Thresholding of statistical maps in functional neuroimaging using the false discovery rate. Neuroimage. 2002;15:870-8.

[26] Helmich RC, Derikx LC, Bakker M, Scheeringa R, Bloem BR, Toni I. Spatial remapping of corticostriatal connectivity in Parkinson's disease. Cerebral cortex (New York, NY : 1991). 2010;20:1175-86.

[27] Hacker CD, Perlmutter JS, Criswell SR, Ances BM, Snyder AZ. Resting state functional connectivity of the striatum in Parkinson's disease. Brain : a journal of neurology. 2012;135:3699-711.

[28] Shine JM, Naismith SL, Lewis SJG. The pathophysiological mechanisms underlying freezing of gait in Parkinson's Disease. Journal of clinical neuroscience : official journal of the Neurosurgical Society of Australasia. 2011;18:1154-7.

[29] Snijders AH, Haaxma Ca, Hagen YJ, Munneke M, Bloem BR. Freezer or non-freezer: clinical assessment of freezing of gait. Parkinsonism \& related disorders. 2012;18:149-54.

[30] Deiber MP, Passingham RE, Colebatch JG, Friston KJ, Nixon PD, Frackowiak RS. Cortical areas and the selection of movement: a study with positron emission tomography. Exp Brain Res. 1991;84:393-402.

[31] Rajput aH, Sitte HH, Rajput a, Fenton ME, Pifl C, Hornykiewicz O. Globus pallidus dopamine and Parkinson motor subtypes: clinical and brain biochemical correlation. Neurology. 2008;70:1403-10. 
[32] Calabresi P, Picconi B, Tozzi A, Ghiglieri V, Di Filippo M. Direct and indirect pathways of basal ganglia: a critical reappraisal. Nat Neurosci. 2014;17:1022-30.

[33] Rothlind JC, York MK, Carlson K, Luo P, Marks WJ, Weaver FM, et al. Neuropsychological changes following deep brain stimulation surgery for Parkinson's disease: comparisons of treatment at pallidal and subthalamic targets versus best medical therapy. J Neurol Neurosurg Psychiatry. 2015;86:622-9.

[34] Fling BW, Cohen RG, Mancini M, Nutt JG, Fair DA, Horak FB. Asymmetric pedunculopontine network connectivity in parkinsonian patients with freezing of gait. Brain. 2013;136:2405-18.

[35] Zhang D, Liu X, Chen J, Liu B, Wang J. Widespread increase of functional connectivity in Parkinson's disease with tremor: a resting-state fMRI study. Frontiers in Ageing Neuroscience. 2015;7:12.

[36] Wu T, Hallett M. The cerebellum in Parkinson's disease. Brain. 2013;136:696-709.

[37] Almeida QJ, Lebold CA. Freezing of gait in Parkinson's disease: a perceptual cause for a motor impairment? J Neurol Neurosurg Psychiatry. 2010;81:513-8.

[38] Dixon ML, Fox KC, Christoff K. Evidence for rostro-caudal functional organization in multiple brain areas related to goal-directed behavior. Brain Res. 2014;1572:26-39.

[39] Blockley NP, Griffeth VE, Stone AJ, Hare HV, Bulte DP. Sources of systematic error in calibrated BOLD based mapping of baseline oxygen extraction fraction. Neuroimage. 2015;122:105-13.

[40] Xu C, Li C, Wu H, Wu Y, Hu S, Zhu Y, et al. Gender differences in cerebral regional homogeneity of adult healthy volunteers: a resting-state FMRI study. Biomed Res Int. 2015;2015:183074.

[41] Hillary FG, Roman CA, Venkatesan U, Rajtmajer SM, Bajo R, Castellanos ND. Hyperconnectivity is a fundamental response to neurological disruption. Neuropsychology. 2015;29:59-75.

[42] Damoiseaux JS, Greicius MD. Greater than the sum of its parts: a review of studies combining structural connectivity and resting-state functional connectivity. Brain structure \& function. 2009;213:525-33. 